AperTO - Archivio Istituzionale Open Access dell'Università di Torino

\title{
La direttiva "rimpatri": la fortezza Europa alza le mura
}

\section{This is the author's manuscript}

Original Citation:

Availability:

This version is available http://hdl.handle.net/2318/87086

since

Terms of use:

Open Access

Anyone can freely access the full text of works made available as "Open Access". Works made available under a Creative Commons license can be used according to the terms and conditions of said license. Use of all other works requires consent of the right holder (author or publisher) if not exempted from copyright protection by the applicable law. 


\title{
La direttiva "rimpatri": la fortezza Europa alza le mura
}

\author{
di Alessandra Algostino
}

(16 luglio 2008)

\section{Il binario unico della fortezza Europa}

II 18 giugno 2008, con 369 voti favorevoli, 197 contrari e 106 astensioni, il Parlamento europeo approva la relazione di Manfred Weber, ovvero il compromesso con il Consiglio sulla direttiva recante Norme e procedure concernenti il rimpatrio di cittadini di paesi terzi soggiornanti illegalmente, la c.d. direttiva rimpatri ${ }^{1}$.

Se qualche merito poteva essere ascritto all'Unione europea, sotto la cui egida si compie in nome della libera concorrenza lo smantellamento dello stato sociale e dei diritti del lavoro ${ }^{2}$, riguardava la disciplina dell'immigrazione, dove la linea guida era la strategia del doppio binario: integrazione e repressione, tutela dei diritti e esigenze di controllo dell'immigrazione ${ }^{3}$.

Si precisa: senza essere con ciò sostenitori di tale strategia. II doppio binario rappresenta già un bilanciamento fra diritti, sanciti dalle costituzioni e dai patti internazionali e continentali sui diritti dell'uomo, e interessi alla gestione dei flussi migratori e al controllo delle frontiere, che facilmente incede in censure di incostituzionalità e in violazione dei patti sui diritti umani. Comunque sia, le direttive comunitarie hanno costituito spesso, soprattutto nei confronti di alcuni Stati, fra i quali I'Italia, facili prede di mistificatrici ed invasive suggestioni securitarie, un argine. Con la c.d. direttiva rimpatri logiche utilitaristiche e invocazioni alla sicurezza esondano anche a livello europeo.

Il nucleo della direttiva, che ha come «campo di applicazione» ${ }^{4}$ «i cittadini di paesi terzi in posizione irregolare nel territorio di uno Stato membro», è costituito dagli artt. 6, 7 e 14, rispettivamente intitolati Decisione di rimpatrio, Allontanamento e

\footnotetext{
${ }^{1}$ II testo qui citato è per l'appunto il testo del compromesso del Parlamento con il Consiglio, ovvero la Relazione Manfred Weber, Norme e procedure comuni concernenti il rimpatrio di cittadini di paesi terzi soggiornanti illegalmente (COM(2005)0391 - C6-0266/2005 - 2005/0167(COD)), Proposta di direttiva, reperibile in www. europarl.europa.eu.

${ }^{2} \mathrm{Ci}$ si permette di rinviare a A. Algostino, Democrazia sociale e libero mercato: Costituzione italiana versus "costituzione europea"?, in Rivista di Diritto Costituzionale, 2007, pp. 98 ss., anche per ulteriori indicazioni bibliografiche, cui adde da ultimo il volume, dal titolo emblematico, di A. Cantaro, I/ diritto dimenticato. Il lavoro nella costituzione europea, Giappichelli, Torino, 2007.

${ }^{3}$ Si leggano ad esempio le Conclusioni della Presidenza al Consiglio europeo di Tampere, 15-16 ottobre 1999 (in www.europarl.europa.eu/summits/tam_it.htm). Le norme europee sono spesso intervenute nel campo dell'immigrazione a fissare delle "norme minime" (si pensi all'accoglienza dei richiedenti asilo o al riconoscimento dello status di rifugiato o, ancora, al ricongiungimento familiare); con la direttiva in esame le norme minime sono sotto il minimo.

${ }^{4}$ Cfr. art. 2, c. 1; al c. 2 si prevede la possibilità per gli Stati membri di non applicare la direttiva nell'ipotesi di respingimento alla frontiera, ovvero di fermo in relazione all'attraversamento della frontiera esterna, o in caso di rimpatrio come sanzione penale, o conseguenza di sanzione penale, o
} 
Trattenimento. "Gli Stati membri prendono una decisione di rimpatrio nei confronti di qualunque cittadino di paesi terzi soggiornante illegalmente sul loro territorio» (art. 6 , par. 1); sono fatte salve alcune possibilità di deroga, sostanzialmente «per motivi caritatevoli, umanitari o di altra natura» (come non pensare alle sanatorie funzionali al mercato del lavoro?), o di sospensione temporanea, quando sia in corso una procedura per il rinnovo del titolo di soggiorno (art. 6, par. 6 ter e 7). È prevista altresì una facoltà di astensione dalla decisione di rimpatrio in presenza di accordi o intese bilaterali, con la possibilità di consegnare I'iirregolare" ad un altro Stato membro, ma in tale ipotesi semplicemente sarà lo Stato membro di consegna ad adottare la decisione di rimpatrio (art. 6, par. 6 bis). La direttiva si premura poi di precisare che il rimpatrio può essere disposto anche contestualmente alla decisione di porre fine al soggiorno regolare (art. 6, par. 8 bis).

Naturale seguito della decisione di rimpatrio è l'allontanamento. È stato introdotto un periodo per la «partenza volontaria» (art. 6 bis), di per sé piuttosto breve (tra 7 e 30 giorni), con possibilità di proroga (par. 2), ma anche con ampie ipotesi di non concessione o riduzione (par. 4), nonché corredato dalla eventuale imposizione di "sanzioni accessorie", quali obbligo di presentazione alle autorità, costituzione di una garanzia finanziaria, consegna dei documenti, obbligo di dimora (par. 3). L'espressione "partenza volontaria» è una mistificazione, o un paradosso, per intendere "ritorno obbligatorio": del resto, nelle definizioni (art. 3), la partenza volontaria è definita come «l'adempimento dell'obbligo di rimpatrio entro il termine fissato a tale scopo nella decisione di rimpatrio».

Se non viene concesso il periodo di partenza volontaria o non viene adempiuto entro tale periodo l'obbligo di rimpatrio, gli Stati membri «adottano [n.d.r.: si noti la perentorietà] tutte le misure necessarie» per eseguire il rimpatrio (art. 7). Fra «tutte le misure necessarie" per allontanare l'iirregolare" rientrano quelle di natura coercitiva (art. 7, par. 3 bis), che un benevolo inciso (di stile?) vorrebbe «in ultima istanza» e che «sono proporzionate e non eccedono un uso ragionevole della forza». Proporzionate e ragionevoli rispetto a cosa? $\dot{E}$ proporzionato e ragionevole trattenere, $\mathrm{o}$, usando un termine più appropriato, detenere una persona perché ha violato norme amministrative concernenti l'ingresso e il soggiorno in un territorio?

Se, infatti, per eseguire il rimpatrio o l'allontanamento non possono essere applicate «altre», non precisate, «misure», gli Stati possono trattenere il rimpatriando, «specie quando» - una parafrasi per dire "non solo"? - sussiste rischio di fuga o il soggetto evita o ostacola rimpatrio o allontanamento, ipotesi, quest'ultima, evidentemente atta a comprendere la maggior parte dei casi (art. 14, par. 1). Proporzione e ragionevolezza delle misure coercitive sussistono quando si discute della privazione del diritto fondamentale alla libertà personale? Quando questa privazione può

in presenza di procedura di estradizione. 
protrarsi per $18 \mathrm{mesi}^{5}$, si può ancora affermare che esiste un diritto alla libertà personale per il cittadino straniero irregolare?

La libertà personale non è l'unica a subire pesanti restrizioni e limitazioni. Si legga l'art. 5 della direttiva: al di là del richiamo al rispetto del principio del non-refoulement, «interesse superiore del minore», «vita familiare» e «condizioni di salute» non devono essere rispettati, ma semplicemente tenuti «nella dovuta considerazione». II binario dei diritti si avvia a grandi passi a divenire un binario morto, a favore di un'alta velocità "espulsiva".

Anche il diritto di chi non ha diritti, l'asilo, è revocato in dubbio dalla direttiva, laddove prevede un «divieto di ingresso» ${ }^{6}$, per cinque anni (art. 9) ${ }^{7}$ : è un divieto revocabile o sospendibile, ma è facile immaginare che di fatto costituirà un ostacolo alle richieste di asilo (per non riferirsi al ricongiungimento familiare) ${ }^{8}$.

Quali diritti restano alla persona umana che è presente illegalmente nel territorio della fortezza Europa? Le clausole generali contenute nella direttiva sulla possibilità di applicare disposizioni più favorevoli (art. 4), nonché il riferimento generico al «rispetto dei diritti fondamentali» (art. 1) ${ }^{9}$ sono smentite dalle puntuali deroghe, ma sarebbe meglio definirle tout court violazioni, dei singoli diritti.

\section{I diritti processuali "speciali" della persona umana "irregolare"}

Salute, diritti alla coesione familiare, tutela del minore, devono dunque essere tenuti nella «dovuta considerazione», la libertà personale viene sacrificata alle esigenze del rimpatrio; sorte non migliore tocca alle garanzie processuali, al diritto di difesa.

Sulla forma dei provvedimenti, si possono segnalare alcuni elementi positivi: le decisioni di rimpatrio e di allontanamento devono essere adottate in forma scritta, motivate in fatto e in diritto e informare sulle modalità di impugnazione (art. 11, par. 1). Meno convincente la previsione della riduzione delle informazioni sui motivi in fatto per esigenze connesse alla sicurezza, alla difesa, a reati (dalla prevenzione al

\footnotetext{
${ }^{5}$ II par. 4 dell'art. 14 stabilisce che il periodo di trattenimento non può superare i sei mesi ed il successivo par. 4 bis ne prevede la proroga per altri dodici mesi.

${ }^{6}$ Le decisioni di rimpatrio sono corredate da un divieto di ingresso «qualora non sia stata concesso un periodo per la partenza volontaria» o «non sia stato ottemperato all'obbligo di rimpatrio», «in altri casi» possono esserlo (art. 9, par. 1).

${ }^{7}$ Il divieto di ingresso «non supera di norma i cinque anni», può superarli in caso di«grave minaccia per l'ordine pubblico, la sicurezza pubblica o la sicurezza nazionale» (art. 9, par. 2).

8 Sottolinea la preoccupazione esposta un recente comunicato di Amnesty International (cfr. www.meltingpot.org/articolo12886.html).

${ }^{9}$ I diritti fondamentali sono citati nell'art. 1 «in quanto principi generali del diritto comunitario e del diritto internazionale»: ora, nulla in contrario a considerare i diritti parte di un patrimonio comune, se ciò ne rafforza 1'efficacia, ma non se si tratta di una operazione che attraverso l'attrazione nel campo dei principi generali rende più vago ed indeterminato sia il loro contenuto sia il loro valore giuridico ( $\mathrm{i}$ diritti sono riconosciuti nelle costituzioni e, spesso sottratti anche, per lo meno nella loro essenza, alla revisione costituzionale).
} 
perseguimento): si tratta di fattispecie di per sé vaghe e, per di più, presumibilmente, adottate da autorità amministrative senza possibilità di controlli effettivi.

Anche riguardo alla traduzione dei provvedimenti, essenziale per l'esercizio concreto del diritto di difesa ${ }^{10}$, la direttiva presenta luci ed ombre: da un lato si prevede la traduzione, pur se «su richiesta» e solo per i «principali elementi» (art. 11, par. 2), dall'altro, si introduce la possibilità di non applicare quanto previsto per chi entra illegalmente e non ha successivamente ottenuto un'autorizzazione (art. 11, par. 2 bis) $)^{11}$.

I diritti della difesa sono poi revocati in dubbio laddove, trattando dei mezzi di ricorso (dinanzi ad una autorità giudiziaria, ma anche amministrativa) avverso le decisioni connesse al rimpatrio (art. 12), si introduce la semplice possibilità di sospensione temporanea del rimpatrio stesso ${ }^{12}$.

Le lacune più gravi nel diritto di difesa si realizzano però nel trattenimento, che avviene «di norma in appositi centri di permanenza temporanea», ma può anche essere in un istituto penitenziario (se pur separatamente dai detenuti ordinari) ${ }^{13}$. immagine concreta della logica che considera l'immigrazione clandestina un reato e criminalizza lo straniero (irregolare e non).

Ciò che colpisce ad una prima lettura sono i tempi per l'intervento dell'autorità giudiziaria: il trattenimento, come si è detto, incide sulla libertà personale e da secoli l'habeas corpus prescrive la pronuncia di un giudice ${ }^{14}$. La direttiva prevede che il trattenimento sia disposto dalle autorità giudiziarie $o$ amministrative ed in quest'ultimo caso un «riesame giudiziario» (art. 14, par. 2), ottemperando alla riserva di giurisdizione generalmente prevista dalle costituzioni in materia di libertà personale ${ }^{15}$, ma senza quei termini brevi e tassativi che della libertà personale costituiscono fondamentale garanzia. La Costituzione italiana, per fare un esempio, stabilisce che in presenza di provvedimenti provvisori adottati dall'autorità di pubblica sicurezza in casi eccezionali di necessità e urgenza sia data entro 48 ore comunicazione all'autorità giudiziaria e che l'eventuale convalida intervenga entro le successive 48 ore (art. 13, c. 3 ); la direttiva ragiona in termini di «pronto riesame»

\footnotetext{
${ }^{10}$ Cfr. Corte costituzionale italiana, sentt. nn. 198 e 227 del 2000.

${ }^{11}$ In tali ipotesi vi sono solo «schede informative generalizzate» che illustrano gli elementi principali del modello uniforme di rimpatrio in almeno cinque fra le lingue più utilizzate.

${ }^{12}$ Incidenter, si noti che anche l'assistenza legale è una «facoltà» (art. 12, par. 3).

${ }^{13}$ Art.15, par. 1.

${ }^{14}$ La Magna Charta del 1215 riconosce il diritto di ciascun uomo libero a non essere «arrestato, imprigionato, privato dei suoi beni, messo fuori della legge, esiliato, molestato in alcuna maniera... se non in virtù di un giudizio legale dei suoi pari e secondo la legge del paese» (art. 39), diritto che - con le dovute cautele - può essere paragonato a quelli sanciti agli articoli 9 («Nessun individuo potrà essere arrestato, detenuto o esiliato arbitrariamente») e 10 («Ogni individuo ha diritto, in posizione di piena uguaglianza, ad una equa e pubblica udienza davanti ad un tribunale indipendente e imparziale, che si pronuncerà sui suoi diritti e i suoi doveri, nonché sulla fondatezza di ogni accusa penale che gli venga rivolta») della Dichiarazione universale dei diritti dell'uomo del 1948.

${ }^{15}$ Vedi Cost. italiana, art. 13.
} 
(art. 14, par. 2), ed anche laddove si tratta della possibilità «su richiesta» di entrare in contatto con rappresentanti legali, familiari e autorità consolari, l'espressione utilizzata è «a tempo debito» (art. 15, par. 2) ${ }^{16}$.

Non è questo però l'unico profilo che mette in discussione l'habeas corpus: a fronte del trattenimento disposto dalle autorità amministrative, gli Stati possono prevedere un «pronto riesame» o semplicemente «il diritto di presentare ricorso per sottoporre la legittimità del trattenimento ad un pronto riesame giudiziario» (art. 14, par. 2). L'intervento del giudice diviene facoltativo, solo a domanda.

La tutela dei diritti umani, nel momento in cui a rivendicarla sono "semplici persone umane" mostra tutte le ambiguità di una proclamazione universale e di un godimento particolare, ovvero legato alla cittadinanza, o, meglio, ad alcune cittadinanze. I diritti universali uguali per tutti cedono il posto alla creazione di un diritto speciale per stranieri, in cui si inscrive la "direttiva rimpatri" e di cui sono espressione chiara e quanto mai preoccupante le varie normative statali c.d. antiterrorismo, nelle quali, fra le tante disposizioni che intaccano - violano - diritti fondamentali, spiccano quelle che introducono o inaspriscono forme di detenzione amministrativa di lungo o indeterminato periodo ${ }^{17}$.

\section{Allontanamento e detenzione nel «superiore interesse del minore»}

II diritto speciale non risparmia nemmeno i minori, con la famiglia o non accompagnati ${ }^{18}$.

L' «interesse superiore del fanciullo deve essere una considerazione preminente» in tutte le decisioni relative ai bambini (art. 3), senza discriminazione alcuna (art. 2), recita la Convenzione sui diritti dell'infanzia adottata dall'Assemblea generale delle Nazioni Unite, New York, il 20 novembre $1989^{19}$ (art. 3); nella direttiva «il prevalente interesse del minore costituisce un criterio fondamentale per il trattenimento dei minori in attesa di allontanamento» (art. 15 bis, par. 5).

La detenzione può costituire, specie nell'ipotesi di minori non accompagnati, un provvedimento nel loro interesse?

\footnotetext{
${ }^{16}$ Anche la previsione del riesame del trattenimento, in caso si prolunghi nel tempo, è indeterminata: il riesame avviene "ad intervalli ragionevoli» (art. 14, par. 3); inoltre pare che non necessariamente esso sia svolto da un giudice, come si desume a contrario dalla precisazione che «nel caso di periodi di trattenimento prolungati, il riesame è sottoposto al controllo di un'autorità giudiziaria».

${ }^{17}$ Si pensi allo status degli enemy combatants negli Stati Uniti, o al fermo di 28 giorni (ma una recente proposta lo vuole prolungare sino a 42 giorni) nel Regno Unito; fra le trattazioni sulle varie discipline antiterrorismo si segnalano, da ultimo, con un approccio comparatistico, P. Bonetti, Terrorismo, emergenza e costituzioni democratiche, il Mulino, Bologna, 2006; M. Tondini, J. P. Pierini, Tavole di legislazione e giurisprudenza comparata sul fenomeno del terrorismo internazionale, in www.forumcostituzionale.it, luglio 2007.

18 Per una prima bibliografia sui minori stranieri, in specie non accompagnati, cfr. il sito http://www.savethechildren.it/minori/bibliografia.htm, cui adde, per un aggiornamento, fra gli altri, G. Campani, O. Salimbeni (a cura di), La fortezza e i ragazzini: la situazione dei minori stranieri in Europa, Angeli, Milano, 2006.

${ }^{19}$ Si ricorda che l'Italia ha ratificato la Convenzione con la legge 27 maggio 1991, n. 176.
} 
La Convenzione di New York contempla la detenzione dei minori come provvedimento di «ultima risorsa» e «dalla durata più breve possibile», oltre che ovviamente rispettoso della riserva di legge (art. 37, lett. b), senza contenere alcun riferimento specifico alla possibilità di detenzione connessa all'immigrazione illegale. La direttiva si limita a ripetere la formula base della Convenzione: «i minori non accompagnati e le famiglie con minori sono trattenuti solo in mancanza di altra soluzione e per un periodo il più possibile breve in funzione delle circostanze» (art. 15 bis, par. 1). Ora, di un' «altra soluzione» non v'è parola nella direttiva, e nella precisazione «in funzione delle circostanze» sembra già di leggere la facile e standardizzata giustificazione del trattenimento. Non dovrebbe essere prevista una procedura specifica, con un esame scrupoloso caso per caso?

E, prima ancora, non vi dovrebbe essere una attenta valutazione dei presupposti per cui l'eventuale rimpatrio e allontanamento è nell'interesse del minore?

La disciplina italiana, se pur non esente da critiche, stabilisce, ad esempio, in primo luogo l'inespellibilità del minore, tranne che per motivi di ordine pubblico e di sicurezza dello Stato ${ }^{20}$; altrimenti il minore lascia il territorio italiano solo come conseguenza del diritto di seguire il genitore o l'affidatario espulsi, ovvero in relazione al rispetto della vita familiare.

Quanto ai minori non accompagnati, dovrebbe essere prevista una attenta valutazione del singolo caso, con opportune indagini nel paese d'origine, ascoltando il minore stesso e seguendolo nel suo reinserimento ${ }^{21}$. L'art. 8 bis, par. 1, della direttiva, nel disciplinare la decisione di rimpatrio dei minori non accompagnati, contempla un'assistenza che tenga «nel debito conto l'interesse superiore del minore»: è diverso dal prevedere - come impone la Convenzione del 1989 un'assistenza basata e finalizzata all'interesse superiore del minore. Si configura una violazione della Convenzione. Volendo ragionare in termini di fonti, prendendo ad esempio l'Italia, si potrebbe bensì osservare che la Convenzione possiede valore di legge ordinaria, disapplicabile, dunque, in presenza di una norma comunitaria confliggente. Non è però difficile argomentare una copertura costituzionale della Convenzione stessa, ovvero rintracciare la preminenza dell' «interesse del fanciullo» nella Costituzione, potendo altresì considerarla alla stregua di un principio fondamentale.

\footnotetext{
${ }^{20}$ Cfr. D. Lgs. $286 / 1998$, artt. 19 , c. 2 , e 31 , c. 4 ; si noti che a decidere in questo caso, a garanzia dell'interesse del minore, è il Tribunale per i minorenni.

${ }^{21}$ La normativa italiana (D. Lgs. 286/1998, art. 33; D.P.C.M. 535/1999) prevede il «rimpatrio assistito» (adottato ed eseguito dal Comitato per i minori stranieri, con il nulla-osta del Tribunale per i minorenni), preceduto da una valutazione mirata e da una indagine nel paese di origine del minore, al fine, in particolare, di garantire il diritto all'unità familiare, adottato, dunque, se risulta concretamente opportuno nell'interesse del minore stesso, che viene sentito, e congiuntamente ad un progetto di reinserimento. Si tratta, peraltro, di una procedura che, al di là delle critiche relative alle sue lacune o agli eccessivi spazi di discrezionalità, è in gran parte rimessa a circolari e, comunque, a fonti sublegislative.
} 
Quanto all'allontanamento vero e proprio (art. 8 bis, par. 2), non vi è alcuna procedura e misura atta a concretizzare l'interesse del fanciullo: la direttiva si limita a stabilire che il minore non accompagnato sia ricondotto, oltre che ad un membro della sua famiglia, a un tutore designato o «presso adeguate strutture di accoglienza nello Stato di ritorno», Stato di ritorno che non è nemmeno necessariamente lo Stato di origine, ma un qualsiasi Stato terzo ${ }^{22}$.

Nell'ipotesi del trattenimento, poi, è stabilito che ai minori non accompagnati è fornita "una sistemazione in istituti dotati di personale e strutture consoni», ma l'inciso precisa: «per quanto possibile» (art. 15 bis, par. 4).

Come nel caso di stranieri che non servono - non rientrano nella manodopera utile, per cui devono essere allontanati e deve essere evitato il rischio che gravitino sulla sanità o sull'assistenza dello Stato "ospitante"23 -, cosi per i minori stranieri la ratio è "liberarsi del problema" il prima possibile, e principi e garanzie, quando vi sono, hanno il netto sapore di formule di stile, imprescindibili in omaggio a carte dei diritti che si vorrebbero esportare ma non si rispettano ${ }^{24}$.

Ma non basta. La direttiva si chiude con la considerazione delle «situazioni di emergenza» (art. 15 ter) che consentono di derogare ai tempi per il riesame giudiziario (prolungandoli), o alle condizioni di trattenimento (anche, ad esempio, a quelle che assicurano una sistemazione separata alle famiglie): il diritto speciale diviene specialissimo?

Nota: il presente intervento sarà pubblicato su Minorigiustizia

\footnotetext{
${ }^{22}$ Non è difficile immaginare che la stipula di accordi bilaterali e comunitari di riammissione sponsorizzata dalla direttiva, in cambio di qualche vantaggio per lo Stato terzo, contempli l'accoglienza presso "strutture adeguate" dello stesso di minori soli.

${ }^{23}$ Emblematiche in proposito le norme che prevedono anche per i cittadini comunitari la disponibilità di «risorse economiche sufficienti» quale condizione per ottenere e mantenere il diritto al soggiorno nel territorio nazionale (e la relativa iscrizione anagrafica) per un periodo superiore a tre mesi, premurandosi di precisare che il cittadino dell'Unione non deve "diventare un onere a carico dell'assistenza sociale dello Stato» e deve possedere un'assicurazione sanitaria o un altro titolo "che copra tutti i rischi nel territorio nazionale» (D. Lgs. 30/2007, spec. artt. 7, 9 e 13, adottato in attuazione della direttiva 2004/38/CE, nonché schema di decreto legislativo in materia di libera circolazione dei cittadini comunitari facente parte del c.d. pacchetto sicurezza).

24 II Presidente della Bolivia, Evo Morales, in una lettera ai governi europei nelle more della discussione sulla "direttiva rimpatrio", afferma che di fronte alla sua approvazione si troveranno «nell'impossibilità etica di approfondire le negoziazioni con l'Unione europea» (fra i molti siti nei quali la lettera si può consultare, cfr. www.meltingpot.org/articolo12872.html).
} 